
\title{
R Reserarch Suare \\ The model for large-scale violence against children surveys in middle schools in Yixing, China
}

\author{
Xiao Yue \\ Yixing CDC \\ Lin Ping \\ Jiangsu CDC \\ Zhang Xujun \\ Southeast University \\ Zheng Aiming \\ Nanjing Medical University \\ Xu Bo \\ Yixing CDC \\ Yaming Yang (D 454369964@qq.com ) \\ Yixing CDC
}

\section{Research article}

Keywords: model, large-scale, violence against children (VAC), surveys, middle schools

Posted Date: February 18th, 2020

DOl: https://doi.org/10.21203/rs.2.23888/v1

License: (c) (1) This work is licensed under a Creative Commons Attribution 4.0 International License.

Read Full License 


\section{Abstract}

Background Aim to build the model for large-scale violence against children (VAC) surveys in middle schools to get comprehensive prevalence data for intervention strategies and activities in Yixing as the only area focus on exploring VAC control and prevention in '2016-2020 children injury prevention program' funded by United Nations International Children's Emergency Fund.

Methods Research protocol and model report underwent rigorous ethical review. Survey teams including investigators, psychological consultants and child health development consultant were recruited and trained in field investigation and intervention, consistently executed seven principle. Crisis cases were defined in full compliance with four criterias. Key considerations for appropriate mode was identified through trial and formal surveys to map crisis cases treatment procedures flow chart.

Results Standard model for large-scale VAC surveys in Yixing had been established, called 'Yixing model', could offer enough psychological protection and guidance for all children in the VAC research. 20863 children participated and occurred different kinds of respondents. 20509 children were general normal and completed their questionnaires. 208 children rejected questionnaries. 3 and 143 crisis cases respectively occurred in field investigation and questionnaries, got intervention according with standard treatment procedures.

Discussion 'Yixing model' was not only participation of investigators and psychological consultants in field investigation, but also extended psychological guidance and intervention from local psychological teams ultimately developing the sustainable intervention mechanism. 'Yixing model' would deserve to promote and replicate in some other related nations or areas.

\section{Background}

Violence against children (VAC) including child maltreatment in all forms (physical, sexual, emotional abuse and neglect) is a widespread issue with far-reaching social and economic consequences[1-4]. In recent years, VAC has received substantial attention, resulting in government initiatives to reduce VAC by strengthening data collection. Media reporting typically focuses on extreme cases, such as death or rape, and collecting reliable data on VAC is a complex undertaking that raises considerable methodological challenges[5]. The researches on VAC are barely beginning in China in recent years. Large-scale survey and comprehensive data on the prevalence of VAC have never been carried out and reported.

Yixing locates in the centre of the Yangtze River Delta Urban Agglomeration in China, has population of over 1.2 million. 44 middle schools with total of about 40000 students were in Yixing in 2018. Yixing has participated in two rounds of programs about injury funded by United Nations International Children's Emergency Fund (UNICEF) in the past ten years[6] and third round '2016-2020 children injury prevention program', as the only area focus on VAC control and prevention in China. We aim to build the model for large-scale VAC measurement surveys in middle school to get comprehensive prevalence data for undertaking intervention strategies and activities. 


\section{Methods}

\section{Ethical approval and consent}

The research was carried out and allowed to publish with permission from the Ethics Committee of Yixing center for disease control and prevention (CDC) (Ref number 2016002) and Ethics Committee of Jiangsu Provincial CDC (Ref number JSJK2018-B025-02). The surveys got informed consents from children's parents or other legal guardians. Children participated could quit at any phase of survey if he or she wanted.

\section{School and Children}

25 of 44 middle schools in Yixing were chosen to paticapate the research by random sampling methods from April 2018 to April 2019. All children in 25 middle schools were field questionnair investigated, while students in third grade senior middle school were excluded. The definition of the Children in the research means human beings below the age of 18 years.

\section{Investigators and psychological consultants}

\section{Psychological support}

Questions involved in field investigation may cause serious emotional fluctuation like anxious and nervous in some children, even excessive behaviors like self-mutilation, has the risk of re-traumatization if ever used to experience impressive violence or existed potential internal psychological trauma. Protecting children from potential dangers due to their participation was the key consideration of research. Professional psychological intervention from Yixing Psychological Association would necessary to protect children in field and continued follow-up investigation. Mental health department of Yixing Renmin Hospital, a Top Grade A hospital accoding to the measures for the administration of the hospital, was designated professional medical institution. Technical supports were got from School of Public Health, Southeast university and Center for Mental Health Education and Research, Nanjing medical university.

Recruit investigator and psychological consultants

86 investigators were public health professionals from Yixing CDC, JiangSu provincial CDC, local governmental hospitals and community health care centers. They had medical background and abundant practical experience of field investigation.

44 psychological consultants from Yixing Psychological Association were recruited in the research. They had qualified license as psychological counselor and more than 5 years counseling experience. 
The teachers of the Institute of Psychology of the Chinese Academy of Science were invited to carry out two stages of professional training and examinations lasting five days directed on VAC to investigators and psychological consultants. Investigator and psychological consultants had qualification of participation following work after passed examinations.

\section{Seven principles}

Seven Principles were strictly executed in the VAC surveys, including relationship principle, control principle, acceptance principle, protection principle, voluntary principle, confidentiality principle and positive principle.

Principle 1: No matter what kinds of symptoms to deal with, top priority was establishing good relationship with children.

Principle 2: Get consent of child at first, let they have senses of control.

Principle 3: Any performance of a child may be normal response in state of stress or psychological trauma, should not to be evaluated and blamed.

Principle 4: Any action has possibility of causing children injuries again should be stopped.

Principle 5: Children have rights to refuse to participate in field investigation and intervention.

Principle 6: All information must be strictly confidential.

Principle 7: All investigation and intervention must end up with positive meaning and guidance.

\section{Questionnaire}

Burden for the collection of information is estimated to average 60 minutes per child, including time for reviewing instruction and answering questionnaire, gathering and maintaining the data.

The questionnaire was made by Center for Chronic Noncommunicable Diseases, Chinese CDC and divided into two parts.

Part 1: Background, information about family demographics, basic economic situation, unattended children and so on.

Part 2: Attitude towards parents or other legal guardians physical punishment, questions about safety issues, sexual violence and multi-angle peer violence, if need psychological help or not and so on.

\section{Identification of crisis case}

The following criterias for identification crisis cases were formulated according to Diagnostic and Statistical Manual of Mental Disorders (version 5) (DSM-5) and International Classification of Diseases 
(version 10) (ICD-10) to recognize the child in need of psychological intervention timely and directly. The child manifested any criteria were defined as a crisis case.

Criteria 1: The child show Intrusions reactions during the investigation: body shaking, shortness of breath or difficulty, could not touch things related to events (touch the scene), not self-forgiving, self-cursing, selfblaming.

Criteria 2: The child show avoidance reactions during the investigation: crying, refusing to answer questions related to violence, physical pain and discomfort, depression, depression, laziness, not communicating with people, request to leave the scene of the investigation.

Criteria 3: The child show high alert reactions during the investigation: fear of strangers and strange environment, cry, break things, beat people, anger, blame, blame.

Criteria 4: The child sought psychological crisis intervention or psychological assistance initiatively or actively in field investigation or his/her questionnaire.

\section{Trial surveys}

Trial surveys in 30 classes in three schools were conducted by experienced psychological consultants and investigators in first one month. The details of questionnaire, difficulties in field investigation, experiences and considerations in trial survey were further summarized and analyzed to improve survey procedures according to cooperation of 18 departments including Yixing CDC and Jiangsu provincial CDC, Yixing Health Commission, Yixing Psychological Association, Yixing Women's Federation, Yixing Education Bureau, Yixing People's Procuratorate, Yixing Civil Affairs Bureau, Southeast University, Nanjing medical university, Yixing Renmin Hospital, etc.

\section{Survey teams}

At least one investigator and one psychological consultant together took charge of one class in field investigation. Another one investigator or psychological consultant was in charge of one teaching building floor. A general leader who was the most experienced investigator and also psychological consultant been trained, called child health development consultant, charged of the investgation and following intervention including contacting with questionnaire crisis cases and overall assessment.

\section{Standard procedures}

In the beginning of field investigaton, child involved in the research got informed consent and the Health Service Information Card including telephone number of relevant organizations of violence control and prevention, such as Yixing Women's Federation, Yixing Women and Chindren's Rights Protection reception center, Youth rights protection and psychological counseling hotline, Women's federation rights protection public welfare hotline, child health development consultant, et, al. 
When the children appeared emotional or behavioral reactions defined as cirsis cases with four criterias during the field investigation, investigators must ensure no body could see their questionnaire during the break to maintain confidentiality, psychological consultants must timely carry out scene psychological intervention following above seven principles to relax their emotions and ask if they would like to make appointment for psychological intervention next time. If the children were still emotionally unstable or unable to continue to answer questions, psychological consultant must ask if they would like to make appointment for answering the questionnaire again or psychological intervention. After consultation intervention, psychological consultants assessed to end case or continue following up. If following up, the survey team decide if referral the case to relevant governmental organizations: Yixing Women's Federation, Yixing Education Bureau, Yixing people's Procuratorate, Yixing Civil Affairs Bureau. Fill out the Referrals Card once make referral and write case report.

When the children defined as crisis cases in their questionnaire, child health development consultant send phone text messgae to them, assess cirsis, assign psychological consultant to psychological consultaion, and decide if make referral.

\section{Results}

Key considerations for developing both methodologically and culturally appropriate VAC surveys was identified through trial surveys and formal investigation, thus standard crisis cases treatment procedures flow chart was mapped. Standard model for large-scale VAC surveys in middle schools in China has been established for the first time, was called 'Yixing model'. The core of 'Yixing model' was protective participation of investigators and psychological consultants in field investigation, following-up psychological intervention and guidance according to the situation of the field evaluation and questionnaire almostly depending on local psychological teams, that would lead to develop the sustainable mechanism to carry out seven strategies for ending VAC (INSPIRE) [7] advocated by WHO. (Figure 1)

20863 children in middle school in Yixing had participated the VAC surveys. 20655 of them had completed the questionnaires, including 20509 normal chidren and 146 crisis cases. 208 children had rejected to answer the questionnaires in field investigation. Response rate of the large-scale VAC surveys was $99 \%$. (Figure 2)

3 crisis cases occured in field investigation and got emergency treatment procedures including scene psychological intervention from psychological consultants and investigators to provide mental protection and ensure sucessfully advance on surveys, appointment of psychological consultation, evaluation of referral and case report.

143 cases had showed willingness to seek help in their questionnaires and been identified as crisis cases. Child health development consultant had contacted of them by phone text message. 5 in 143 questionnaire crisis cases had replied and got emergency treatment procedures including appointment of psychological consultation intervention, evaluation of referral and case report. 


\section{Conclusion}

There were no ready-made model for VAC survey or intervention to adopt before we started '2016-2020 children injury prevention program'. We explored feasible and acceptable modes of large-scale VAC surveys and interventions following, built the treat procedures of crisis casesbeen the first time in China. The core of 'Yixing model' was not only participation of investigators and psychological consultants in field investigation, but also extended psychological intervention from local psychological teams ultimately developing the sustainable intervention mechanism in line with INSPIRE. Comprehensive and accurate prevalence data had got for intervention strategies and activities in the future. Experience of ${ }^{\prime}$ Yixing model' would deserve to promote and replicate in some other related nations or areas over the world.

Violence discipline at home remains a largely unacknowledged form of VAC, although corporal punishment in educational settings was prohibitten in China now. 146 crisis cases have occured in the large-scale violence surveys, that is less frequent than we expected, but not means with minor impact. In part, VAC remains hidden because of current social background, such as physical punishment at home and bullying peers, often socially accepted or tacitly condoned. Reluctance on the part of victims to report their incidents poses a challenge to exposing the true extent and nature of VAC.

Only 5 in 143 crisis cases intended to ask for help in their questionnaries have replied and participate psychological intervention, that may because of normally reticent culture tradition of Chinese, widely perceived as a social taboo, relate to the level of trust of children, maturity of intervention means of school bullying, suggest ensuring that violence in all its forms is documented through solid data is a first step towards its elimination, general violence-related children would through a long way of from think to seek help to cope with the experience or take action to protect themselves from further victimization.

\section{Abbreviations}

United Nations International Children's Emergency Fund UNICEF Violence against children VAC center for disease control and prevention CDC

\section{Declarations}




\section{Ethics approval and consent to participate}

The research was established and carried out with permission from the Ethics Committee of Yixing CDC (Ref number 2016002) and Ethics Committee of Jiangsu Provincial CDC (Ref number JSJK2018-B02502). Written informed consent was obtained from individual or guardian participants.

\section{Consent for publication}

Not applicable.

\section{Availability of data and material}

All data analysed during this study are included in this published article.

\section{Competing interests}

The authors declare no potential conflicts of interest with respect to the research, authorship and publication of this article. All authors have read and agreed on its content and are accountable for all aspects of the accuracy and integrity of the manuscript.

\section{Funding}

We wish to thank UNICEF China, Jiangsu provincial CDC and Yixing CDC for the financial supports.

\section{Authors' contributions}

YYM designed the research. $X Y, L P, Z A M, Z X J, X B$ and YYM participated the exploring of the model for large-scale violence against children surveys in Yixing middle schools. XY wrote the report.

\section{Acknowledgements}

We appreciated Yixing Psychological Association for psychological intervention in investigation to protect children.

\section{References}

1. World report on child injury prevention. 2008, World Health Organization, 164-167.

2. A familiar face Violence in the lives of children and adolescents. November 2017, United Nations International Children's Emergency Fund (UNICEF), 13-15.

3. Shonkoff JP, Garner AS. The lifelong effects of early childhood adversity and toxic stress. Pediatrics 2012, 129(1), 232-246. 
4. The contagious spread of violence among US adolescents through social networks. AJPH research February 2017, 107(2), 288-294.

5. Fallon B, Trocme N, Fluke J, MacLaurin B, Tonmyr L, Yuan YY: Methodological challenges in measuring child maltreatment. Child Abuse Negl 2010, 34(1), 70-79.

6. Injury intervention work in Yixing was exchanged at the summary meeting of the ministry of health's injury control index research project. Yixing center for disease control and prevention network, 2013, (http://www.yxcdc.com/jkxx/2576.jhtml)

7. INSPIRE: seven strategies for ending violence against children. World Health Organization, 2017. (https://www.who.int/violence_injury_prevention /violence/inspire/en/)

\section{Figures}




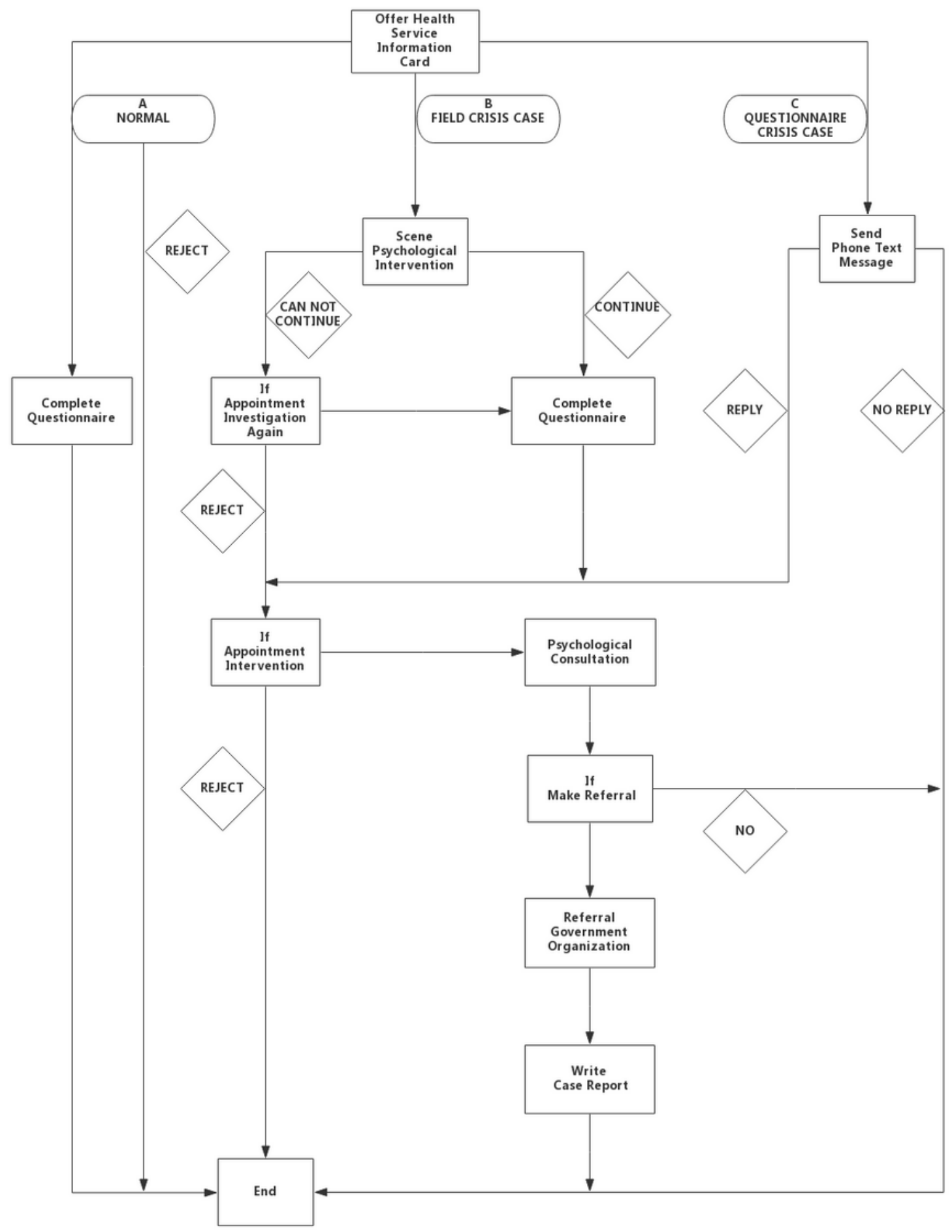

\section{Figure 1}

Emergency treatment procedures flow chart All children involved got Health Service Information Cards first. They were divided into A, B, C population becuse of different conditions in field investigation and questionnaires. Treatment procedures were carried out from top to bottom following the arrows. Comment in rectangle represented one treatment procedure. Comment in rhombus represented different situations of crisis cases. "End" represented the end of the VAC survey and intervention of all children. 


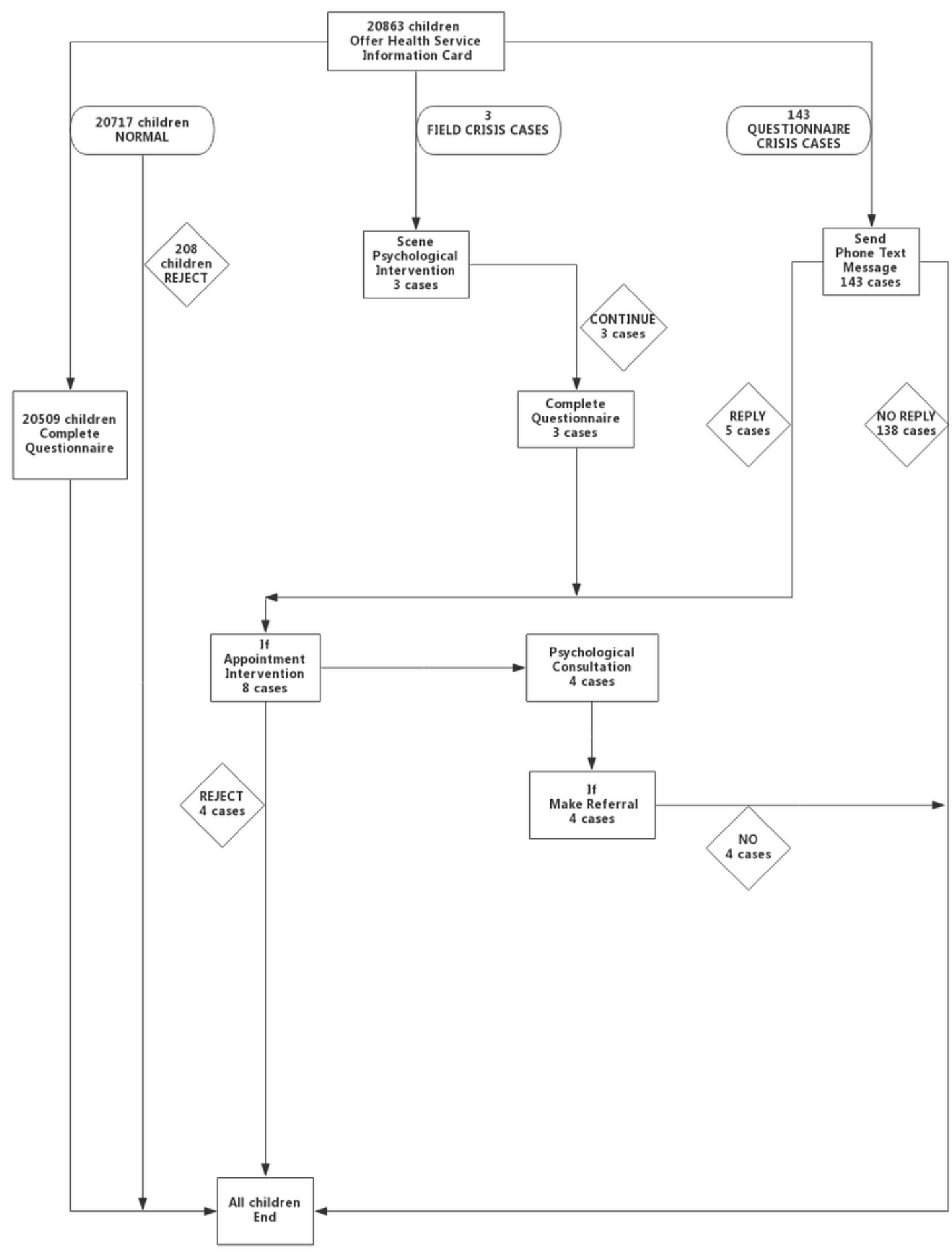

\section{Figure 2}

Number of different kinds of crisis cases 20863 children in middle school in Yixing got Health Service Information Cards. Numbers of children of different situations and procedures were showed in rectangles and rhombuses. Emergency treatment procedures that no one experienced were temporally removed to simplify figure, including "appointment investigation again", "refeeral government organization", "write case report". 